\section{Dwarf Bermudagrass Responses to Flurprimidol and Paclobutrazol}

\author{
Patrick E. McCullough ${ }^{1}$ \\ Department of Plant Biology and Pathology, Rutgers, The State University of \\ New Jersey, New Brunswick, NJ 08901-8520 \\ Haibo Liu' and Lambert B. McCarty ${ }^{3}$ \\ Department of Horticulture, Clemson University, Clemson, SC 29634-0375
}

Additional index words. plant growth regulators, root growth, TifEagle, Champion

\begin{abstract}
Plant growth regulators are applied to inhibit uneven shoot growth of putting green turf but research is limited on responses of dwarf-type bermudagrass cultivars to growth inhibition. Experiments were conducted at the Clemson University Greenhouse Complex with 'Champion' and 'TifEagle'bermudagrass grown in polyvinylchloride containers with 40 cm depths and $177 \mathrm{~cm}^{2}$ areas built to United States Golf Association specification. Flurprimidol was applied at $0.14,0.28$, and $0.48 \mathrm{~kg} \cdot \mathrm{ha}^{-1} \mathrm{a}$.i. and paclobutrazol at $0.14 \mathrm{~kg} \cdot \mathrm{ha}^{-1} \mathrm{a}$.i. on

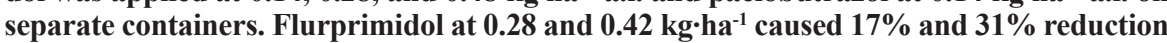
in turf color 5 weeks after treatment (WAT), respectively. 'Champion' exhibited unacceptable turf injury $(>30 \%) 2$ WAT from paclobutrazol and all flurprimidol rates. 'TifEagle' had unacceptable turf injury from flurprimidol at $0.42 \mathrm{~kg}^{\circ} \mathrm{ha}^{-1} 2 \mathrm{WAT}, 0.28 \mathrm{~kg} \cdot \mathrm{ha}^{-1} 3 \mathrm{WAT}$, and $0.14 \mathrm{~kg}^{\circ} \mathrm{ha}^{-1} 4 \mathrm{WAT}$ that did not recover. Moderate injury $(16 \%$ to $30 \%)$ was observed from paclobutrazol on 'TifEagle' but ratings were acceptable. After 6 weeks, flurprimidol at $0.14,0.28$, and $0.42 \mathrm{~kg} \cdot \mathrm{ha}^{-1}$ reduced bermudagrass green shoot density (GSD) per square centimeter by $20 \%, 40 \%$, and $40 \%$, respectively, while paclobutrazol reduced GSD $12 \%$. 'TifEagle' total clipping yield was reduced $60 \%, 76 \%$, and $86 \%$ from flurprimidol at 0.14 , 0.28 , and $0.42 \mathrm{~kg} \cdot \mathrm{ha}^{-1}$, respectively, and $37 \%$ from paclobutrazol. 'Champion' total clipping yield was reduced $82 \%, 90 \%$, and $90 \%$ from flurprimidol at $0.14,0.28$, and $0.42 \mathbf{~ k g}^{\circ} \mathbf{h a}^{-1}$, respectively, and $58 \%$ from paclobutrazol. After 6 weeks, flurprimidol reduced 'Champion' total root mass by $44 \%$ over all three rates. 'Champion' treated with paclobutrazol had similar total root mass to the untreated. 'TifEagle' treated with all PGRs had similar rooting to the untreated. Overall, flurprimidol will likely not be suitable for dwarf bermudagrass maintenance at these rates; however paclobutrazol may have potential at $\leq 0.14 \mathbf{~ k g} \cdot \mathbf{h a}^{-1}$. Chemical names used: Flurprimidol $\{\alpha-(1-m e t h y l e t h y l)-\alpha-[4-($ trifluoro-methoxy) phenyl] 5-pyrimidine-methanol\}; Paclobutrazol, $(+/-)-\left(\mathbf{R}^{*}, \mathbf{R}^{*}\right)-[\beta-[(4-\mathrm{chlorophenyl})$ methyl]- $\alpha-(1$, 1-dimethyl)-1H-1,2,4,-triazole-1-ethanol.
\end{abstract}

Plant growth regulators (PGRs) have become important tools for turf managers to inhibit uneven shoot growth and promote uniform golf course putting green surfaces. PGRs modify turfgrass growth by inhibiting either cell division or cell elongation. Phytotoxicity and reduced root growth have prevented widespread use of cell division inhibitors; therefore, gibberellic acid (GA) inhibitors are more frequently used because of less likelihood for leaf burn and turfgrass injury (Murphy et al., 2005).

Two popular GA inhibitors, paclobutrazol and flurprimidol, inhibit the conversion of entkaurene to ent-kaurenoic acid by blocking the enzyme ent-kaurene oxidase (Sponsel, 1995; Buchanan etal., 2000). Ent-kaurene oxidase is a membrane-bound cytochrome $\mathrm{P} 450$ monooxygenase enzyme with a heme prosthetic group responsible for the oxidation of ent-kaurene, ent-kaurenol, and ent-kaurenal forming $\mathrm{GA}_{12}$ aldehyde (Sirvastava, 2002). Paclobutrazol, a

Received for publication 8 June 2004. Accepted for publication 10 Oct. 2004. Contribution from the Clemson Univ. Agricultural Experiment Station. ${ }^{1}$ Program associate.

${ }^{2}$ Associate professor. To whom reprint requests should be addressed; e-mail haibol@clemson.edu. ${ }^{3}$ Professor. triazole compound, inhibits P-450 monooxygenase enzymes involved in brassinosteroid and GA syntheses. Flurprimidol contains a pyrimidine group and has similar activity as a GA and sterol synthesis inhibitor.

Existing concerns with PGR applications include turfgrass discoloration and root growth inhibition. Flurprimidol and paclobutrazol are effective PGRs for common and hybrid bermudagrass (Cynodon dactylon $\times C$. transvaalensis) maintained at golf course fairway mowing heights (Johnson, 1992); however, turf injury has been observed with single and repeated applications on these grasses (Johnson, 1989, 1990, 1992, 1994). Dwarf-type bermudagrasses are hybrid cultivars introduced for golf course putting greens that may be more sensitive to these PGRs due to finer leaf textures and lower growth habits. Research is currently lacking to verify this.

Dwarf-type bermudagrass cultivars tolerate long term mowing heights of $3.2 \mathrm{~mm}$ or closer (McCarty and Miller, 2002). Routine closer mowing heights of dwarf cultivars, such as 'Champion' and 'TifEagle', may reduce root vigor, depth, and mass compared to traditional bermudagrass cultivars (White, 1998). Dwarf-type bermudagrasses have also shown sensitivity to PGR and herbicide applications (McCullough, 2004; McCullough et al., 2004), raising concerns over PGR induced root growth restrictions.

Registered rates of paclobutrazol on creeping bentgrass, 0.28 and $0.42 \mathrm{~kg} \cdot \mathrm{ha}^{-1}$, have shown unacceptable turf injury $(>30 \%)$ on 'TifEagle' (McCullough et al., 2005). Similar results were observed on 'TifEagle' with repeated applications of flurprimidol at 0.42 $\mathrm{kg} \cdot \mathrm{ha}^{-1}$ (McCullough etal, 2004). Applications of paclobutrazol at $0.14 \mathrm{~kg} \cdot \mathrm{ha}^{-1}$ per 6 weeks have shown to produce similar turf quality to untreated 'TifEagle' but root mass was reduced after 12 weeks. Root mass reductions of $43 \%$ were also observed in these experiments with repeated applications of flurprimidol at 0.42 $\mathrm{kg} \cdot \mathrm{ha}^{-1}$ per 3 weeks on 'TifEagle'.

Although adjusted PGR rates may provide acceptable dwarf bermudagrass shoot quality, inhibited root growth may prove their application unacceptable for routine maintenance. Single applications of these compounds at appropriate rates may be more suitable for dwarf bermudagrass if turf quality and root growth are unaffected. Although paclobutrazol rates have been tested on dwarf bermudagrass, research is limited on various flurprimidol rates. The objective of this greenhouse experiment was to investigate growth responses of 'Champion' and 'TifEagle' treated with flurprimidol at $0.14,0.28$, and $0.42 \mathrm{~kg} \cdot \mathrm{ha}^{-1}$ and paclobutrazol at $0.14 \mathrm{~kg} \cdot \mathrm{ha}^{-1}$.

\section{Materials and Methods}

Two studies were conducted over 6 weeks from September to October 2003 (Study 1) and November to December 2003 (Study 2) at the Clemson University Greenhouse Complex, Clemson, S.C. Greenhouse day/night temperatures were set at about $26 / 20^{\circ} \mathrm{C}$. Due to reduced natural lighting during winter months, supplemental lighting was added for about 3 $\mathrm{h} \cdot \mathrm{d}^{-1}$ at $50 \mu \mathrm{mol} \cdot \mathrm{m}^{-2} \cdot \mathrm{s}^{-1}$ and the experimental design was a randomized complete block with three replications. Plugs were collected from 'TifEagle' and 'Champion' greens located in the Turf Service Center, Clemson, established in July 2002 and 2003, respectively. Bermudagrass plugs were washed free of soil and roots were cut about $2.5 \mathrm{~cm}$ from the thatch layer, and then transplanted to polyvinyl chloride containers. Containers were built approximately to United States Golf Association specifications (USGA Green Section Staff, 1993) to help mimic field conditions. An 85:15(v/v) sand and peat moss rootzone mix was the soil medium consisting of $6 \%$ coarse sand $(0.5$ to $1.0 \mathrm{~mm})$, $30 \%$ medium sand ( 0.25 to $0.5 \mathrm{~mm}$ ), $48 \%$ fine sand ( 0.1 to $0.25 \mathrm{~mm})$, and $16 \%$ very fine sand $(<0.1 \mathrm{~mm})$. Containers had $40 \mathrm{~cm}$ depths with $177 \mathrm{~cm}^{2}$ surface areas and had drainage holes cut at about $6 \mathrm{~mm}$ diameter at the bottom. Starting fertilizer, 9N-18 $\mathrm{P}_{2} \mathrm{O}_{5}-17 \mathrm{~K}_{2} \mathrm{O}$ (Scott's, Marysville, Ohio) was mixed into the soil at $48 \mathrm{~kg} \cdot \mathrm{ha}^{-1} \mathrm{~N}$.

Bermudagrass was irrigated and mowed with grass sheers (Black and Decker, Towson, Md.) at $4 \mathrm{~mm} 5 \mathrm{~d} \cdot$ week $^{-1}$ and had a 3-week establishment period to resume active growth before initial PGR treatments. Nitrogen as ammonium nitrate solution was applied at 12 
Table 1. Turf color for 'Champion' and 'TifEagle' bermudagrass treated with paclobutrazol and flurprimidol in two combined greenhouse studies. Different letters indicate a significant difference at the 0.05 probability level by column.

\begin{tabular}{lccccccc}
\hline Plant & & \multicolumn{5}{c}{ Week after treatment } \\
\cline { 2 - 7 } $\begin{array}{l}\text { growth } \\
\text { regulator }\end{array}$ & $\begin{array}{c}\text { Rate } \\
\left(\mathrm{kg} \cdot \mathrm{ha}^{-1} \mathrm{a} \text { a.i. }\right)\end{array}$ & 1 & 2 & 3 & 4 & 5 & 6 \\
\cline { 2 - 7 } & --- & $7.0 \mathrm{~b}$ & $7.0 \mathrm{a}$ & $7.0 \mathrm{bc}$ & $7.0 \mathrm{bc}$ & $7.0 \mathrm{a}$ & $7.0 \mathrm{ab}$ \\
Untreated & 0.14 & $7.2 \mathrm{ab}$ & $7.2 \mathrm{a}$ & $7.5 \mathrm{a}$ & $7.4 \mathrm{ab}$ & $7.1 \mathrm{a}$ & $7.2 \mathrm{a}$ \\
Flurprimidol & 0.28 & $7.2 \mathrm{ab}$ & $7.2 \mathrm{a}$ & $6.9 \mathrm{c}$ & $6.9 \mathrm{c}$ & $5.8 \mathrm{~b}$ & $6.8 \mathrm{~b}$ \\
Flurprimidol & 0.42 & $7.3 \mathrm{a}$ & $7.0 \mathrm{a}$ & $7.2 \mathrm{abc}$ & $6.3 \mathrm{~d}$ & $4.8 \mathrm{c}$ & $5.9 \mathrm{c}$ \\
Flurprimidol & 0.14 & $7.3 \mathrm{a}$ & $7.1 \mathrm{a}$ & $7.4 \mathrm{ab}$ & $7.5 \mathrm{a}$ & $7.0 \mathrm{a}$ & $7.2 \mathrm{a}$ \\
Paclobutrazol & & &
\end{tabular}

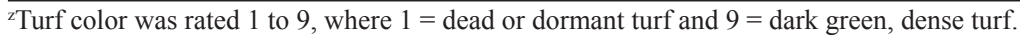

Table 2. Turf injury for 'Champion' and 'TifEagle' bermudagrass treated flurprimidol and paclobutrazol in two combined greenhouse experiments. Different letters indicate a significant difference at the 0.05 probability level for a cultivar by column.

\begin{tabular}{|c|c|c|c|c|c|c|c|}
\hline \multirow{3}{*}{$\begin{array}{l}\text { Plant } \\
\text { growth } \\
\text { regulator }\end{array}$} & \multirow{3}{*}{$\begin{array}{c}\text { Rate } \\
\left(\mathrm{kg} \cdot \mathrm{ha}^{-1} \text { a.i. }\right)\end{array}$} & \multicolumn{6}{|c|}{ Week after treatment } \\
\hline & & \multicolumn{6}{|c|}{ Turf injury ${ }^{z}(\%)$} \\
\hline & & 1 & 2 & 3 & 4 & 5 & 6 \\
\hline \multicolumn{8}{|c|}{ Champion bermudagrass } \\
\hline Untreated & --- & $0 \mathrm{c}$ & $0 \mathrm{~b}$ & $0 \mathrm{~d}$ & $0 \mathrm{~d}$ & $0 \mathrm{~d}$ & $0 \mathrm{~d}$ \\
\hline Flurprimidol & 0.14 & $13 \mathrm{bc}$ & $50 \mathrm{a}$ & $43 \mathrm{~b}$ & $60 \mathrm{~b}$ & $43 \mathrm{~b}$ & $42 \mathrm{~b}$ \\
\hline Flurprimidol & 0.28 & $30 \mathrm{a}$ & $50 \mathrm{a}$ & $55 \mathrm{ab}$ & $68 \mathrm{ab}$ & $71 \mathrm{a}$ & $73 \mathrm{a}$ \\
\hline Flurprimidol & 0.42 & $25 \mathrm{ab}$ & $58 \mathrm{a}$ & $63 a$ & $80 \mathrm{a}$ & $76 \mathrm{a}$ & $80 \mathrm{a}$ \\
\hline Paclobutrazol & 0.14 & $23 \mathrm{ab}$ & $45 \mathrm{a}$ & $28 \mathrm{c}$ & $30 \mathrm{c}$ & $22 \mathrm{c}$ & $17 \mathrm{c}$ \\
\hline \multicolumn{8}{|c|}{ TifEagle bermudagrass } \\
\hline Untreated & --- & $0 \mathrm{~b}$ & $0 \mathrm{c}$ & $0 \mathrm{~b}$ & $0 \mathrm{~d}$ & $0 \mathrm{c}$ & $0 \mathrm{~d}$ \\
\hline Flurprimidol & 0.14 & $7 \mathrm{ab}$ & $7 \mathrm{bc}$ & $17 \mathrm{a}$ & $32 \mathrm{bc}$ & $13 \mathrm{c}$ & $27 \mathrm{c}$ \\
\hline Flurprimidol & 0.28 & $7 \mathrm{ab}$ & $17 \mathrm{~b}$ & $37 \mathrm{a}$ & $47 \mathrm{ab}$ & $50 \mathrm{ab}$ & $57 \mathrm{~b}$ \\
\hline Flurprimidol & 0.42 & $13 \mathrm{a}$ & $40 \mathrm{a}$ & $44 \mathrm{a}$ & $62 \mathrm{a}$ & $65 \mathrm{a}$ & $72 \mathrm{a}$ \\
\hline Paclobutrazol & 0.14 & $3 \mathrm{~b}$ & $3 \mathrm{bc}$ & $7 \mathrm{~b}$ & $17 \mathrm{~cd}$ & $23 \mathrm{bc}$ & $3 d$ \\
\hline
\end{tabular}

Table 3. Green shoot density after 6 weeks for 'Champion' and 'TifEagle'bermudagrass treated with flurprimidol and paclobutrazol in two combined greenhouse experiments. Different letters indicate a significant difference at the 0.05 probability level.

\begin{tabular}{lccc}
\hline $\begin{array}{l}\text { Plant } \\
\text { growth } \\
\text { regulator }\end{array}$ & $\begin{array}{c}\text { Rate } \\
\left(\mathrm{kg} \cdot \mathrm{ha}^{-1} \mathrm{a} . \mathrm{i} .\right)\end{array}$ & Cultivar & $\begin{array}{c}\text { Green } \\
\text { shoots/ } \\
\mathrm{cm}^{2}\end{array}$ \\
\hline Untreated & --- & & $25 \mathrm{a}$ \\
Flurprimidol & 0.14 & & $20 \mathrm{~b}$ \\
Flurprimidol & 0.28 & & $15 \mathrm{c}$ \\
Flurprimidol & 0.42 & $15 \mathrm{c}$ \\
Paclobutrazol & 0.14 & $22 \mathrm{~b}$ \\
& & & \\
& \multicolumn{3}{c}{$\begin{array}{c}\text { Champion } \\
\text { TifEagle }\end{array}$} \\
& \multicolumn{3}{c}{$21 \mathrm{~b}$} \\
\hline
\end{tabular}

$\mathrm{kg} \cdot \mathrm{ha}^{-1} \cdot$ week $^{-1}$. Flurprimidol (50WP) was applied at $0,0.14,0.28$, and $0.42 \mathrm{~kg} \cdot \mathrm{ha}^{-1}$ and paclobutrazol (2SC) at $0.14 \mathrm{~kg} \cdot \mathrm{ha}^{-1}$ in a greenhouse spray chamber (model SB6-094; Devries Manufacturing (Hollandale, Minn.) delivering $720 \mathrm{~L} \cdot \mathrm{ha}^{-1}$. Flurprimidol rates were chosen based on registered rates for 'Tifgreen' (Sepro Corp., 2002) and previous research on 'TifEagle'.

Turf color was rated visually on a 1 to 9 scale with 9 being dark green turf and 1 completely dormant turf. Ratings were based on leaf color and below 7 were considered unacceptable. Turf injury was evaluated weekly on a percent scale basis, where $0=$ no injury, $1 \%$ to $15 \%=$ minor discoloration, $16 \%$ to $30 \%=$ moderate injury, $>30 \%=$ unacceptable injury, and $100 \%=$ completely dead turf. Injury ratings were based on loss of stand density and turf uniformity relative to untreated turf. Green shoot density counts per square centimeter were made after 6 weeks in three random samples per experimental

color Cultivarby PGR interaction was not detected for turf color; therefore treatment effects were pooled across cultivar and studies. Turf color was enhanced $4 \% 1$ WAT by flurprimidol at $0.42 \mathrm{~kg} \cdot \mathrm{ha}^{-1}$ and paclobutrazol (Table 1). However, flurprimidol at 0.28 and $0.42 \mathrm{~kg} \cdot \mathrm{ha}^{-1}$ caused a $17 \%$ and $31 \%$ reduction in turf color by 5 WAT, respectively. Other PGR treatments produced similar turf color to the untreated.

Turfinjury. Cultivar $\times$ PGR interaction was detected for turf injury; therefore, results are presented by cultivar (Table 2). 'Champion' exhibited unacceptable turf injury 2 WAT from paclobutrazol and all flurprimidol rates. 'Champion' injury recovered to acceptable levels from paclobutrazol by 5 WAT. 'TifEagle' had unacceptable turf injury from flurprimdol at $0.42 \mathrm{~kg} \cdot \mathrm{ha}^{-1} 2 \mathrm{WAT}, 0.28 \mathrm{~kg} \cdot \mathrm{ha}^{-1}$ 3 WAT, and $0.14 \mathrm{~kg} \cdot \mathrm{ha}^{-1} 4$ WAT. Moderate injury was observed from paclobutrazol on 'TifEagle' but ratings were acceptable. Bermudagrass injury from flurprimidol applications did not recover by 6 WAT.

Cultivar $\times$ PGR interaction was not detected for green shoot density after 6 weeks; therefore, results were pooled over cultivar and PGR (Table 3). After 6 weeks, flurprimidol at $0.14,0.28$, and $0.42 \mathrm{~kg} \cdot \mathrm{ha}^{-1}$ reduced bermudagrass green shoot density by $20 \%$, $40 \%$, and $40 \%$, respectively. Turf treated with paclobutrazol had $12 \%$ reduced green shoot density than non-PGR treated turf. Averaged across PGR treatments, 'TifEagle' green shoot density was $11 \%$ greater than 'Champion'.

Clipping yield. . Cultivar $\times$ PGR interaction was detected for weekly clipping yield; therefore results are presented by cultivars. PGR treatments reduced clipping yield about $70 \%$ for both bermudagrass cultivars 1 WAT (Table 4). 'TifEagle' treated with paclobutrazol had clipping yield inhibited from 1 to 4 WAT but clipping yield was similar to untreated turf 5 WAT. 'Champion' clippings were suppressed by paclobutrazol $74 \% 3$ WAT with $45 \%$ reductions by 6 WAT. Total clipping yield was reduced $60 \%, 76 \%$, and $86 \%$ from flurprimidol at $0.14,0.28$, and $0.42 \mathrm{~kg} \cdot \mathrm{ha}^{-1}$, respectively, and $37 \%$ from paclobutrazol on 'TifEagle'. Total clipping yield was reduced $82 \%, 90 \%$, and $90 \%$ from flurprimidol at $0.14,0.28$, and $0.42 \mathrm{~kg} \cdot \mathrm{ha}^{-1}$, respectively, and $58 \%$ from paclobutrazol on 'Champion'.

Root mass and length. Cultivar $\times$ PGR interaction was detected for root mass and length and thus cultivars are presented separately. Comparing untreated turf, 'TifEagle' averaged $66 \%$ less total root mass and $33 \%$ less root length than 'Champion' (Table 5). Flurprimidol reduced 'Champion' total root mass $44 \%$ over all three rates. Flurprimdiol at 0.28 and $0.42 \mathrm{~kg} \cdot \mathrm{ha}^{-1}$ reduced 'Champion' root mass $43 \%$ at the 0 to $10 \mathrm{~cm}$ soil depth and $61 \%$ at the 10 to $20 \mathrm{~cm}$ soil depth compared to the untreated. 'Champion' treated with paclobutrazol had reduced root mass at the 20 to $30 \mathrm{~cm}$ soil depth but total root mass was similar to the untreated. 'TifEagle' treated with PGRs had similar rooting to the untreated.

\section{Discussion}

Plant growth regulators are commonly applied to golf course putting greens to reduce uneven shoot growth and enhance turf quality. Ideally, PGRs should inhibit turfgrass shoot growth without turf injury or deleterious effects on root growth. These principles have even greater importance for PGR selection on fine textured dwarf bermudagrasses with potentially reduced root growth from closer mowing heights.

'Champion' and 'TifEagle' displayed unacceptable turf injury from flurprimidol with little to no recovery after 6 weeks. Paclobutrazol injured 'Champion' more severely than 'TifEagle'. Plugs collected for the 'TifEagle' were established in the field 1 year before 'Champion' and observed injury on 'Champion', relative to 'TifEagle', may have resulted 
Table 4. Dry clipping yield for 'Champion' and 'TifEagle' bermudagrass treated with flurprimidol and paclobutrazol in two combined greenhouse experiments. Different letters indicate a significant difference at the 0.05 probability level for a cultivar by column.

\begin{tabular}{|c|c|c|c|c|c|c|c|c|}
\hline \multirow{2}{*}{$\begin{array}{l}\text { Plant } \\
\text { growth } \\
\text { regulator }\end{array}$} & \multirow{2}{*}{$\begin{array}{c}\text { Rate } \\
\left(\mathrm{kg} \cdot \mathrm{ha}^{-1} \text { a.i. }\right)\end{array}$} & \multicolumn{7}{|c|}{ Clipping yield $\left(\mathrm{g} \cdot \mathrm{m}^{-2}\right)$} \\
\hline & & \multicolumn{7}{|c|}{ Week after treatment } \\
\hline \multicolumn{9}{|c|}{ Champion bermudagrass } \\
\hline Flurprimidol & 0.28 & $0.5 \mathrm{~b}$ & $0.3 \mathrm{c}$ & $0.2 \mathrm{~b}$ & $0.2 \mathrm{~b}$ & $0.0 \mathrm{c}$ & $0.0 \mathrm{c}$ & $1.2 \mathrm{c}$ \\
\hline Flurprimidol & 0.42 & $0.5 \mathrm{~b}$ & $0.4 \mathrm{bc}$ & $0.2 \mathrm{~b}$ & $0.1 \mathrm{~b}$ & $0.0 \mathrm{c}$ & $0.0 \mathrm{c}$ & $1.2 \mathrm{c}$ \\
\hline Paclobutrazol & 0.14 & $1.0 \mathrm{~b}$ & $1.0 \mathrm{ab}$ & $0.5 \mathrm{~b}$ & $0.4 \mathrm{~b}$ & $0.9 \mathrm{~b}$ & $1.2 \mathrm{~b}$ & $5.0 \mathrm{~b}$ \\
\hline Flurprimidol & 0.14 & $0.9 \mathrm{c}$ & $0.6 \mathrm{c}$ & $0.9 \mathrm{c}$ & $0.4 \mathrm{c}$ & $1.5 \mathrm{~b}$ & $1.6 \mathrm{a}$ & $5.9 \mathrm{c}$ \\
\hline Flurprimidol & 0.28 & $0.9 \mathrm{c}$ & $0.6 \mathrm{c}$ & $0.5 \mathrm{~d}$ & $0.5 \mathrm{c}$ & $0.5 \mathrm{c}$ & $0.5 \mathrm{~b}$ & $3.5 \mathrm{~cd}$ \\
\hline Flurprimidol & 0.42 & $0.6 \mathrm{c}$ & $0.5 \mathrm{c}$ & $0.3 \mathrm{~d}$ & $0.2 \mathrm{c}$ & $0.2 \mathrm{c}$ & $0.2 \mathrm{~b}$ & $2.0 \mathrm{~d}$ \\
\hline Paclobutrazol & 0.14 & $1.4 \mathrm{~b}$ & $1.0 \mathrm{~b}$ & $1.2 \mathrm{~b}$ & $1.2 \mathrm{~b}$ & $2.4 \mathrm{a}$ & $2.0 \mathrm{a}$ & $9.2 \mathrm{~b}$ \\
\hline
\end{tabular}

Table 5. Root length and dry root mass after six weeks for 'Champion' and 'TifEagle' bermudagrass treated with flurprimidol and paclobutrazol in two combined greenhouse studies.

\begin{tabular}{|c|c|c|c|c|c|c|c|c|c|c|c|}
\hline \multirow{2}{*}{$\begin{array}{l}\text { Plant } \\
\text { growth } \\
\text { regulator }\end{array}$} & \multirow{2}{*}{$\begin{array}{c}\text { Rate } \\
\left(\mathrm{kg} \cdot \mathrm{ha}^{-1} \text { a.i. }\right)\end{array}$} & \multicolumn{5}{|c|}{ Champion } & \multicolumn{5}{|c|}{ TifEagle } \\
\hline & & $\begin{array}{l}\text { Root length } \\
(\mathrm{cm})\end{array}$ & \multicolumn{4}{|c|}{ Root mass $\left(\mathrm{g} \cdot \mathrm{m}^{-2}\right)$} & $\begin{array}{l}\text { Root length } \\
(\mathrm{cm})\end{array}$ & \multicolumn{4}{|c|}{ Root mass $\left(\mathrm{g} \cdot \mathrm{m}^{-2}\right)$} \\
\hline Untreated & --- & $26.7 \mathrm{aA}^{\mathrm{z}}$ & $14.3 \mathrm{abA}$ & $2.8 \mathrm{aA}$ & $0.5 \mathrm{aA}$ & $17.6 \mathrm{aA}$ & $18.0 \mathrm{aB}$ & $5.5 \mathrm{aB}$ & $0.4 \mathrm{aB}$ & $0.0 \mathrm{aB}$ & $5.9 \mathrm{aB}$ \\
\hline Flurprimidol & 0.28 & $19.9 \mathrm{bA}$ & $8.2 \mathrm{cA}$ & $1.0 \mathrm{bcA}$ & $0.1 \mathrm{bA}$ & $9.3 \mathrm{bA}$ & $16.3 \mathrm{aA}$ & $5.1 \mathrm{aB}$ & $0.3 \mathrm{aB}$ & $0.0 \mathrm{aA}$ & $5.4 \mathrm{aB}$ \\
\hline Flurprimidol & 0.42 & $20.3 \mathrm{bA}$ & $8.1 \mathrm{cA}$ & $1.2 \mathrm{bcA}$ & $0.2 \mathrm{abA}$ & $9.5 \mathrm{bA}$ & $15.2 \mathrm{aB}$ & $4.6 \mathrm{aB}$ & $0.4 \mathrm{aA}$ & $0.0 \mathrm{aA}$ & $5.0 \mathrm{aB}$ \\
\hline Paclobutrazol & 0.14 & $23.3 \mathrm{abA}$ & $15.5 \mathrm{aA}$ & $2.1 \mathrm{abA}$ & $0.2 \mathrm{bA}$ & $17.8 \mathrm{aA}$ & $18.2 \mathrm{aA}$ & $4.8 \mathrm{aB}$ & $0.8 \mathrm{aB}$ & $0.0 \mathrm{aA}$ & $5.6 \mathrm{aB}$ \\
\hline
\end{tabular}

${ }^{2}$ Lower case letters are mean separations among columns, capital letters are mean separations between cultivars by row and parameter.

from differences in PGR absorbency potential. Nevertheless, both cultivars exhibited injury following PGR applications.

'Champion' bermudagrass showed severe root mass and root length reductions from flurprimidol applications while 'TifEagle' was unaffected. Differences observed between cultivars resulted from greater root growth of 'Champion' relative to 'TifEagle'. White (1998) noted 'TifEagle' had 57\% less root mass than 'Champion' 4 months after sprigging the cultivars. Thus, the more actively growing roots of 'Champion' may have been more vulnerable to flurprimidol induced restrictions compared to 'TifEagle'.

Paclobutrazol injured both grasses; however, recovery occurred to acceptable levels with generally no root growth restrictions. Of the two PGRs, paclobutrazol may have potential for applications at lower rates, such as 0.05 to $0.10 \mathrm{~kg} \cdot \mathrm{ha}^{-1}$, on dwarf bermudagrasses. Future research is needed on these PGRs, but flurprimidol will likely not be suitable for dwarf bermudagrass maintenance at traditional putting green application rates.

\section{Literature Cited}

Buchanan, B.B., W. Gruissem, and R.L. Jones. 2000 Biochemistry and molecular biology of plants. Amer. Soc. Plant Physiol., Rockville, Md.

Johnson, B.J. 1989. Response of bermudagrass (Cynodon spp.) to plant growth regulators. Weed Technol. 3:440-444.

Johnson, B.J. 1990. Response of bermudagrass (Cynodon spp.) cultivars to multiple plant growth regulator treatments. Weed Technol. 4:549-554.

Johnson, B.J. 1992. Response of Tifway bermudagrass to rate and frequency of flurprimidol and paclobutrazol application. HortScience 27(3):230-232.

Johnson, B.J. 1994. Influence of plant growth regulators and mowing on two bermudagrasses. Agron. J. 86:805-810.

McCarty, L.B. and G.L. Miller. 2002. Managing bermudagrass turf: Selection, construction, cultural practices and pest management strategies, p. 7 . Sleeping Bear Press, Chelsea, Mich.

McCullough, P.E. 2004. Physiological response of 'TifEagle' bermudagrass to nitrogen and trinexapac-ethyl, p. 39-44. MS thesis. Clemson Univ., Clemson, S.C.

McCullough, P.E., H. Liu, L.B. McCarty, and T. Whitwell. 2004 Response of TifEagle' bermudagrass to seven plant growth regulators. HortScience
39:1759-1762.

McCullough, P.E., H. Liu, L.B. McCarty, and T. Whitwell. 2005. Physiological response of 'TifEagle' bermudagrass to paclobutrazol. HortScience 40:224-226.

Murphy, T.R., T. Whitwell, B. McCarty, and F.H. Yelverton. 2005. Turfgrass plant growth regulators, p. 705-714. In: L.B. McCarty (ed.). Best golf course management practices. 2nd ed. Prentice-Hall, Upper Saddle River, N.J.

Sepro Corp. 2002. Cutless 50W Label, p. 3. Sepro Corp. Carmel, Ind.

Sirvastava, L.M. 2002. Plant growth and development, hormones and environment. Academic Press, San Diego, Calif.

Sponsel, V.M. 1995. The biosynthesis and metabolism of gibberellins in higher plants, p. 66-97. In: P.J. Davies (ed.). 1995. Plant hormones: Physiology, biochemistry, and molecular biology. 2nd ed. Kluwer Academic Publ., Dordrecht, The Netherlands.

United States Golf Association (USGA) Green Section Staff. 1993. USGA recommendations for a method of putting green construction. The 1993 Revision. USGA Green Sect. Record 31(2):1-3.

White, R.H. 1998. Performance and management of new dwarf bermudagrasses. Texas A \& M Univ., Texas Agr. Expt. Sta. 1998 Semi-annual Res. Progr. Rpt. 